\title{
Opinion
}

\section{Plant-Pathogen Interactions: Underestimated Roles of Phyto-oxylipins}

Estelle Deboever ${ }^{1,2, *}$

estelle.deboever@doct.uliege.be

\section{Magali Deleu ${ }^{1}$}

Sébastien Mongrand $^{3}$

Laurence Lins ${ }^{1,4}$

Marie-Laure Fauconnier ${ }^{2}, 4$

${ }^{\mathbf{1}}$ Molecular Biophysics at Interface Laboratory (LBMI), Gembloux Agro-Bio Tech, University of Liège, 2, Passage des Déportés, B-5030 Gembloux, Belgium

${ }^{2}$ Laboratory of Natural Molecules Chemistry (LCMN), Gembloux Agro-Bio Tech, University of Liège, 2, Passage des Déportés, B-5030 Gembloux, Belgium

${ }^{3}$ Laboratory of Membrane Biogenesis (LBM), Research Mix Unity (UMR) 5200, National Scientific Research Center (CNRS), University of Bordeaux, Bordeaux, France

${ }^{*}$ Correspondence

${ }^{\mathbf{4}}$ These authors contributed equally to this work and must be considered as co-last authors



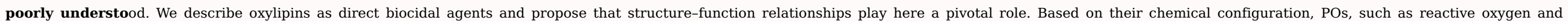

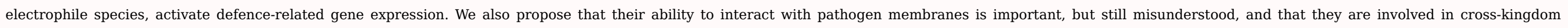

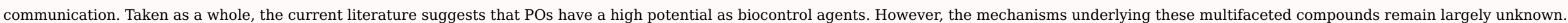

\section{Oxylipin Basics}

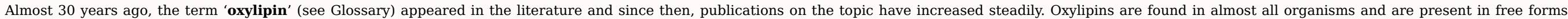

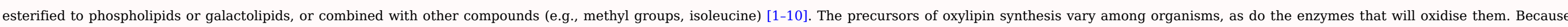

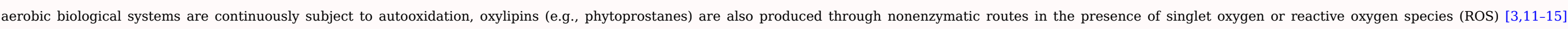

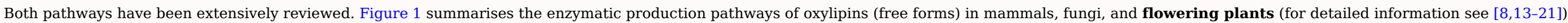




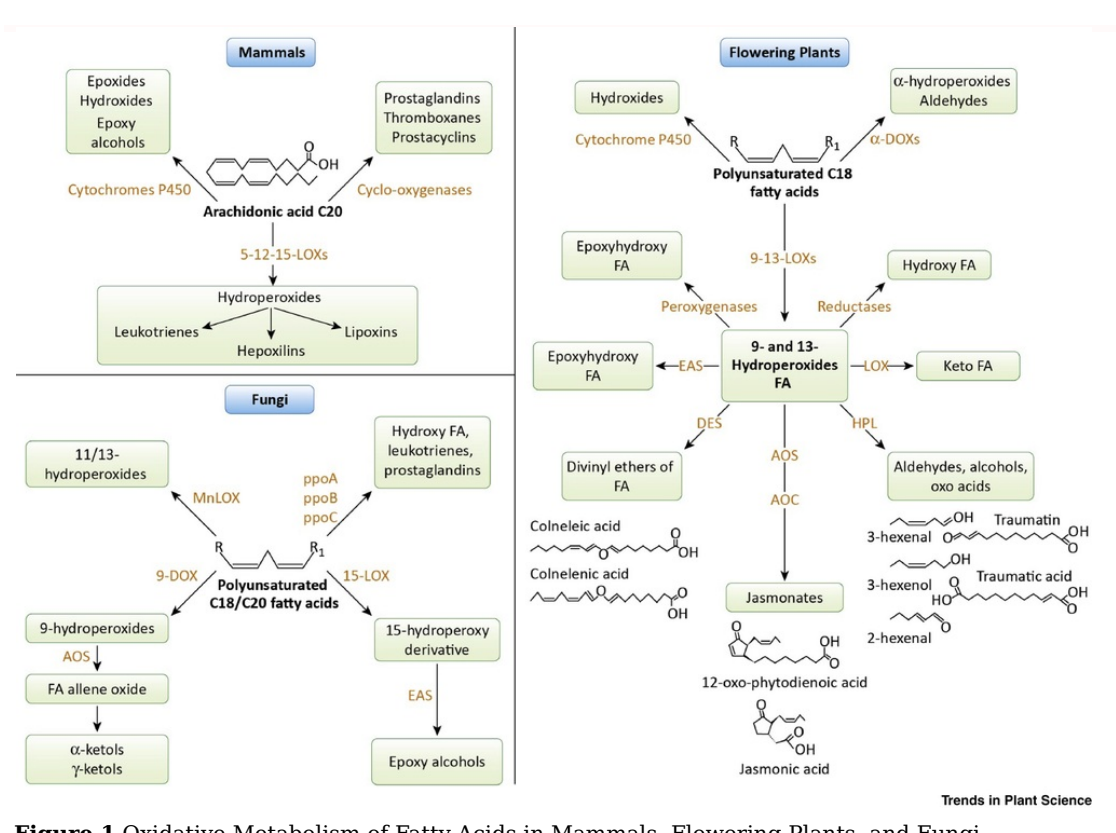

Figure 1 Oxidative Metabolism of Fatty Acids in Mammals, Flowering Plants, and Fungi.

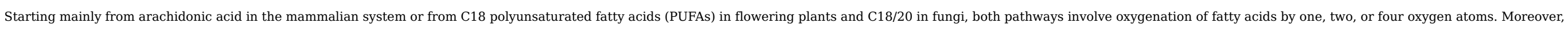

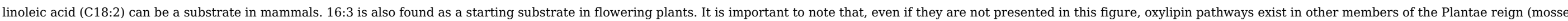

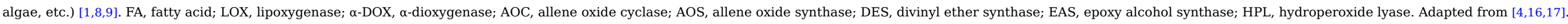

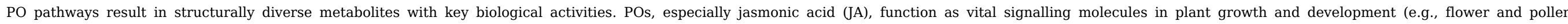

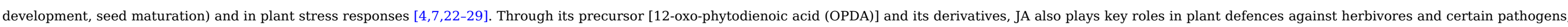
mainly necrotrophic $[25,29,30]$. Accordingly, this may be extended to all POs playing crucial roles in early defence reactions against insect or pathogen attacks.

\section{Shaping the PO Signature}

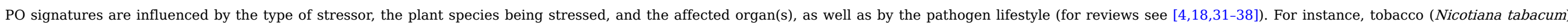

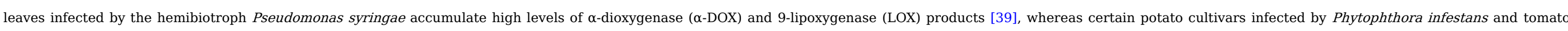
leaves infected by necrotrophic Botrytis cinerea accumulate 9(S)- and 13(S)-polyunsaturated fatty acid (FA) (PUFA) hydroperoxides (HPOs) [40-42].

Considering the context of this review and the extensive diversity among oxylipins and pathogens, we have decided to focus on the role of POs in the interactions between host plants and their pathogens.

\section{Effectiveness of POs as Signalling Molecules in Plant Defence Mechanisms}

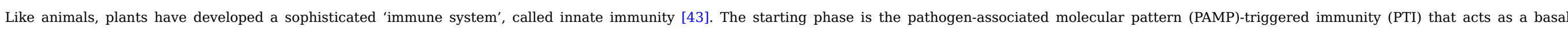


resistance (SAR) is finally activated, which will keep the plant alarmed and prepared for other attacks for weeks to months [44]. SAR can also be activated by elicitors [45-48].

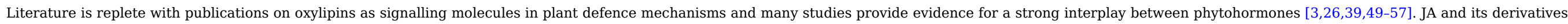








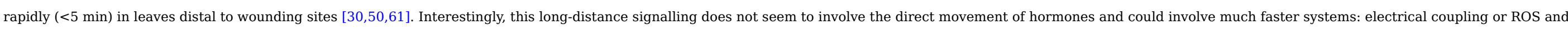

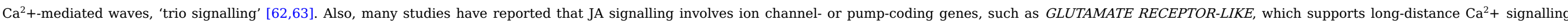

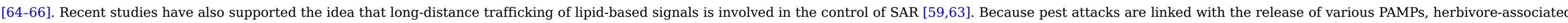

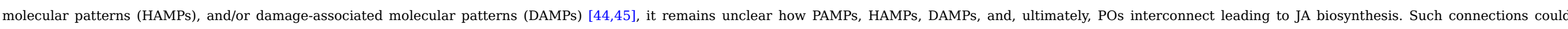

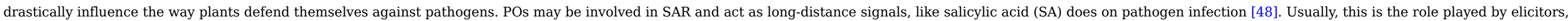
suggesting that POs may represent a new source of eliciting molecules.

\section{Study of PO: Mind the Gap}

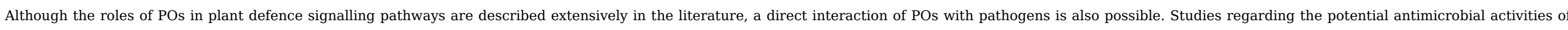

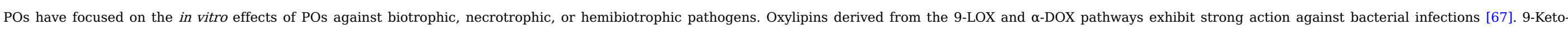

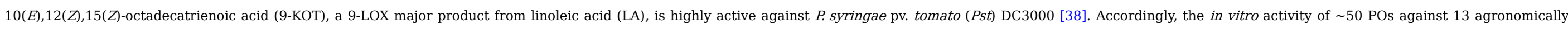

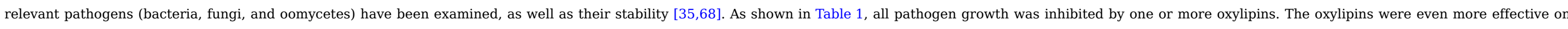

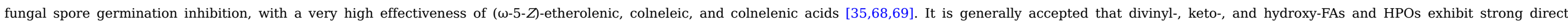
antimicrobial activities while others, JA and some volatile aldehydes, seem to be only implied in signalling.

Table 1 Summary of the Most Effective Antimicrobial Free POs (Based on [35,67], In Vitro Assays) ${ }^{\mathrm{a}, \mathrm{b}}$

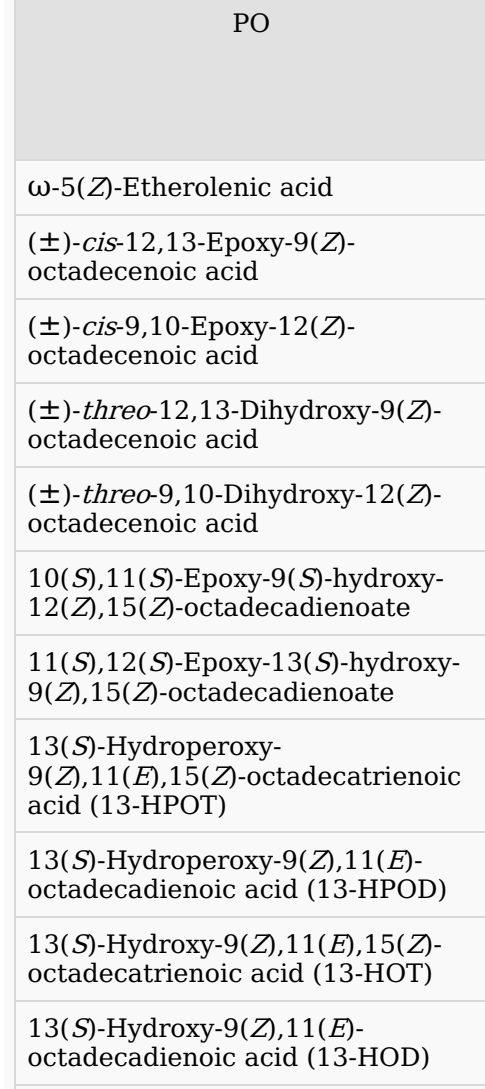

Strain

Bacteria

Pc

Ps

$\mathrm{Xc}$

$\mathrm{Ab}$

$\mathrm{Bc}$

$+++\quad-$

$++$

$-\quad+\quad+$

$++$

$-$

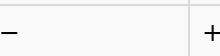

$-2$

\begin{tabular}{l|l|l|} 
& -2 & + \\
++ & - & + \\
++ & - & -
\end{tabular}

$-$

$-\quad++$

\begin{tabular}{|l|l|l|}
- & - & - \\
- & + & - \\
- & - & - \\
- & + & -
\end{tabular}

\begin{tabular}{l|l|l|}
- & $\mathrm{x}$ & - \\
- & $\mathrm{x}$ & + \\
- & ++ & ++++
\end{tabular}

\begin{tabular}{l|l}
- & - \\
- & + \\
- & -
\end{tabular}

++
-
+

\begin{tabular}{|l|l|}
- & - \\
+ & - \\
\hline++++ & ++++ \\
\hline- & ++++ \\
\hline+++ & ++++ \\
\hline+ & ++++
\end{tabular}

$++$

\begin{tabular}{|c|c|c|c|c|c|}
\hline Lm & Rsp & Ss & $\mathrm{Vl}$ & $\mathrm{Pi}$ & $\mathrm{Pp}$ \\
\hline & - & - & - & + & - \\
\hline
\end{tabular}


13-Keto-9( $Z), 11(E), 15(Z)$ octadecatrienoic acid (13-KOT)

9(S)-Hydroperoxy-

$10(E), 12(Z), 15(Z)-$
octadecatrienoic acid (9-HPOT)

9(S)-Hydroperoxy-10(E),12(Z)octadecadienoic acid (9-HPOD)

9(S)-Hydroxy-10(E),12(Z),15(Z)octadecatrienoic acid (9-HOT)

9(S)-Hydroxy-10(E),12(Z)-

octadecadienoic acid (9-HOD)

9-Keto-10( $E), 12(Z)$

octadecadienoic acid (9-KOD)

9-Keto-10( $E), 12(Z), 15(Z)$

octadecatrienoic acid (9-KOT)

Colneleic acid

Colnelenic acid

Anacardic acid

12-Oxo-10,15(Z)-phytodienoic acid (OPDA)

2(E)-Nonenal

3(Z)-Nonenal

2(E)-Hexenal

3(Z)-Hexenal

\begin{tabular}{|c|c|c|c|c|c|c|c|c|c|c|c|c|}
\hline- & - & - & - & - & - & - & $\mathrm{x}$ & - & $\mathrm{x}$ & $\mathrm{x}$ & - & + \\
\hline- & + & - & - & ++ & +++ & - & $\mathrm{x}$ & + & $\mathrm{x}$ & $\mathrm{x}$ & + & ++++ \\
\hline- & - & - & - & +++ & ++++ & - & - & - & - & - & - & ++++ \\
\hline- & + & - & - & ++ & ++++ & - & $\mathrm{x}$ & - & $\mathrm{x}$ & $\mathrm{x}$ & - & ++++ \\
\hline- & - & - & - & ++ & +++ & ++ & $\mathrm{x}$ & + & $\mathrm{x}$ & $\mathrm{x}$ & - & ++++ \\
\hline- & - & - & - & - & ++++ & + & $\mathrm{x}$ & - & $\mathrm{x}$ & $\mathrm{x}$ & - & ++++ \\
\hline- & ++ & +++ & - & - & +++ & - & $\mathrm{x}$ & - & $\mathrm{x}$ & $\mathrm{x}$ & - & +++ \\
\hline- & + & - & - & - & ++++ & - & $\mathrm{x}$ & - & $\mathrm{x}$ & $\mathrm{x}$ & - & ++++ \\
\hline- & ++++ & - & - & - & ++++ & + & $\mathrm{x}$ & - & $\mathrm{x}$ & $\mathrm{x}$ & - & - \\
\hline- & ++ & - & - & + & ++++ & - & $\mathrm{x}$ & - & $\mathrm{x}$ & $\mathrm{x}$ & + & + \\
\hline- & - & ++++ & - & - & - & + & $\mathrm{x}$ & - & $\mathrm{x}$ & $\mathrm{x}$ & +++ & - \\
\hline- & + & - & + & +++ & ++++ & + & $\mathrm{x}$ & + & $\mathrm{x}$ & $\mathrm{x}$ & + & ++++ \\
\hline- & ++ & - & - & - & - & - & $\mathrm{x}$ & - & $\mathrm{x}$ & $\mathrm{x}$ & + & - \\
\hline- & ++ & - & - & - & - & + & $\mathrm{x}$ & ++ & $\mathrm{x}$ & $\mathrm{x}$ & - & - \\
\hline $\mathrm{x}$ & ++++ & ++++ & ++++ & ++++ & ++++ & ++++ & $\mathrm{x}$ & ++++ & $\mathrm{x}$ & $\mathrm{x}$ & ++++ & ++++ \\
\hline- & ++ & - & - & - & - & - & $\mathrm{x}$ & - & $\mathrm{x}$ & $\mathrm{x}$ & - & - \\
\hline
\end{tabular}

a The oxylipins were tested at concentrations of around $100 \mu \mathrm{M}$ and measurements were taken after 24-h incubation.

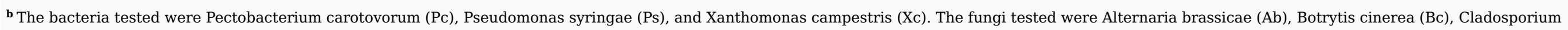

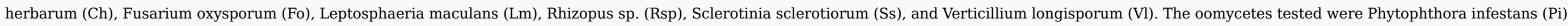
and Phytophthora parasitica (Pp). ++++ , very highly effective; +++ , highly effective; ++ , moderately effective; + , effective; - , not effective; $\mathrm{x}$, not tested.



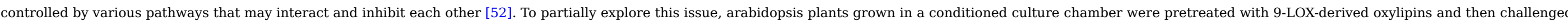

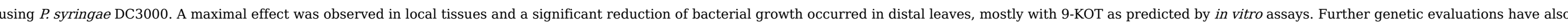

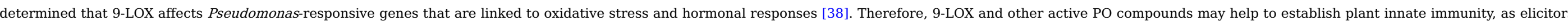

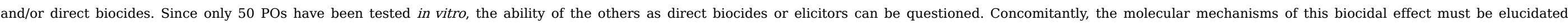

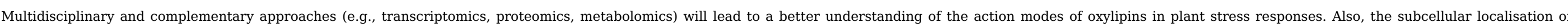

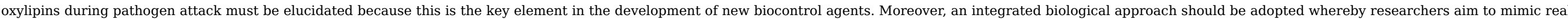
field stresses (culture substrate, choice of strain, mode of application, duration and timing of stress, growing conditions, etc.).

\section{The Road to the Next Level}




\section{The Link Between Structure and Biocidal Activity}

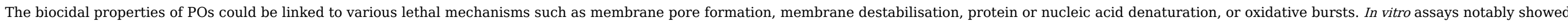

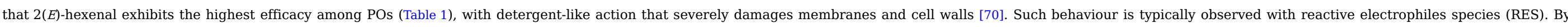

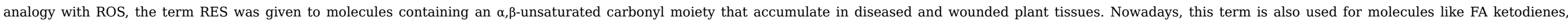



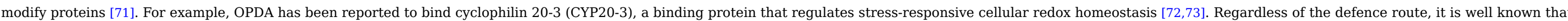
the excessive production of electrophilic molecules can disrupt natural cellular functions and, eventually, cause cell collapse [74].

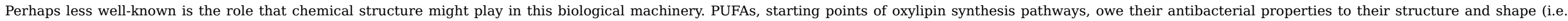

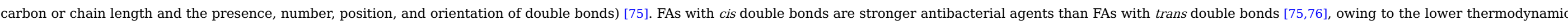


against pathogens than monounsaturated FA [77].



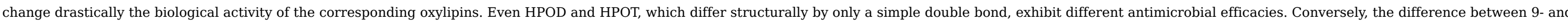

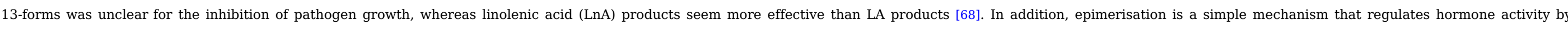
converting bioactive (+)-7-iso-jasmonoyl-L-isoleucine into the inactive form, JA-Ile [30].

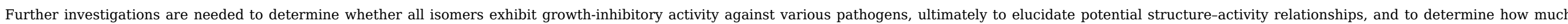

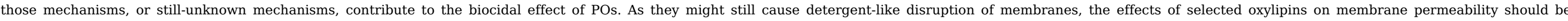
investigated in the future. Nevertheless, they presumably depend on the chemical nature of the oxylipin, the damage exerted, and the molecular mechanisms involved.

\section{Are Membranes Active Actors or Passive Filters in PO Biocidal Activity?}



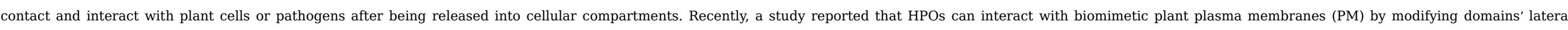

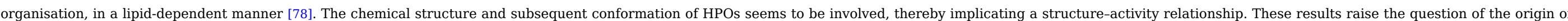
PO antimicrobial activities and whether/how POs interact with the pathogen PM (Figure 2). 
(1) Exogenous application of PO

on infected plants and/or

endogenous production of

PO by infected plants

(2)

Perceptio

Perception
mechanisms

mechanisms

Plant Plasma Membrane



plant plasma membrane?

pathogen plasma membran

Activation of specific Modification of the

defence genes?
membrane fluidity?

(3) Defence response

\section{Trends in Plant Science}

Figure 2 Hypothetical Schematic Diagram Showing the Roles of the Plasma Membrane (PM) in Plant Oxylipin (PO)-Pathogen Interactions.

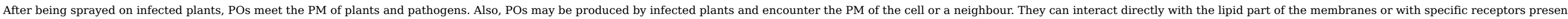



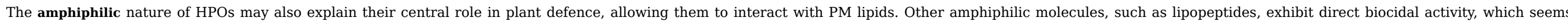

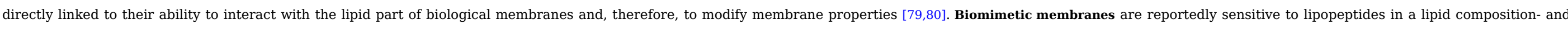

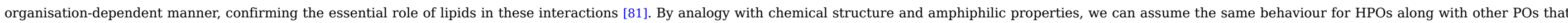
interact with bacterial or fungal membranes.





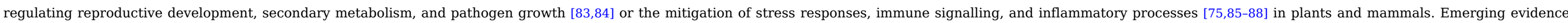

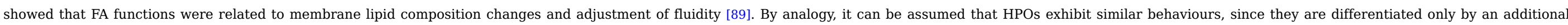





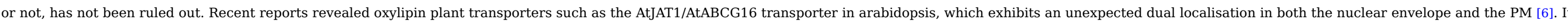

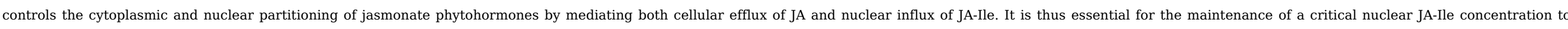

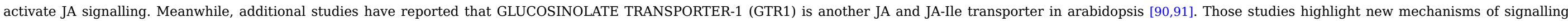
hormone regulation, and many other transporters may be described in coming years. 


\section{The Parallelism with Eicosanoids}

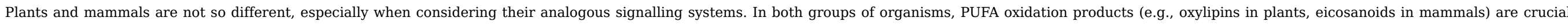

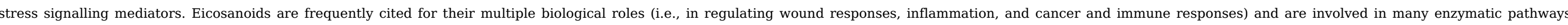
(Figure 1).

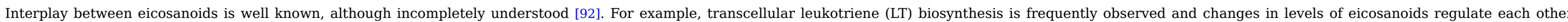

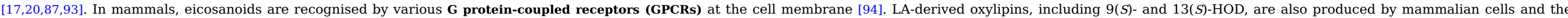
human GPCR G2A functions as their specific receptor [17,95-97]. This suggests a possible involvement of GPCRs in HPO recognition in plant cells.




communication and applications.

\section{Oxylipin Interkingdom ‘Communication’: An Even More Complicated Interplay?}

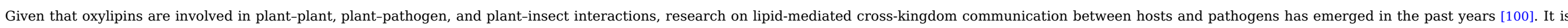
becoming increasingly clear that organisms commonly use oxylipin pathways as a means of communication to elicit biological responses.



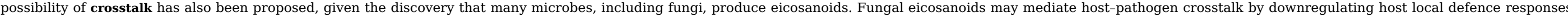

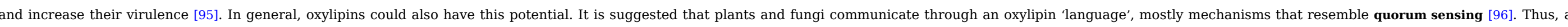



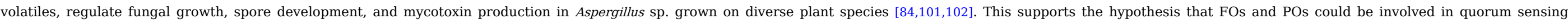



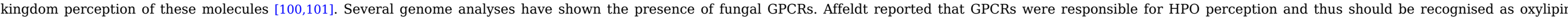
receptors [96,97]. This reinforces the hypothesis that POs, along with all forms of stimuli, might be perceived by fungi through a GPCR-mediated cascade (Figure 3). 


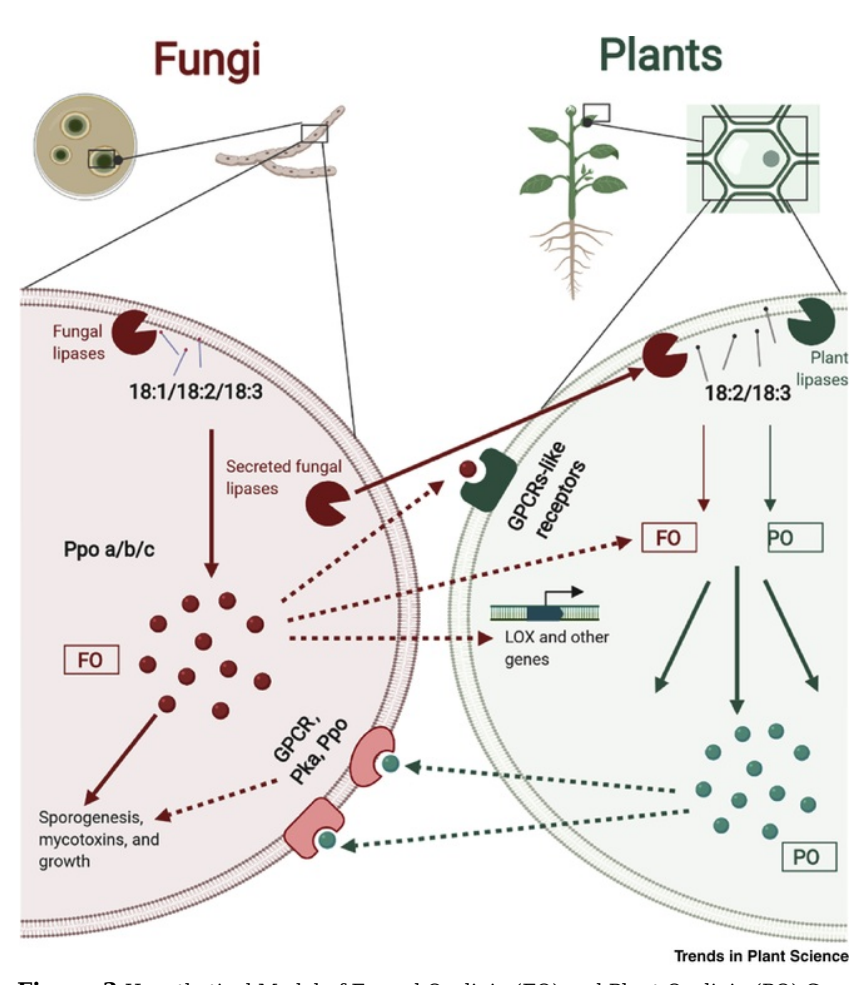

Figure 3 Hypothetical Model of Fungal Oxylipin (FO) and Plant Oxylipin (PO) Crosstalk (Based on [16,37,99]).



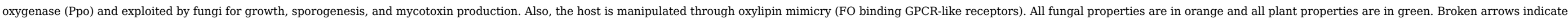
hypothetical interactions and unbroken arrows established interactions.

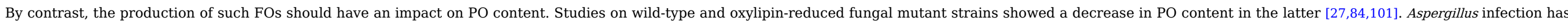

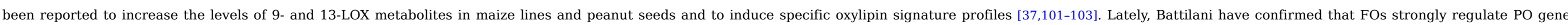



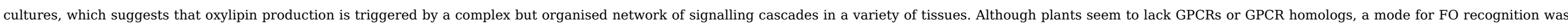

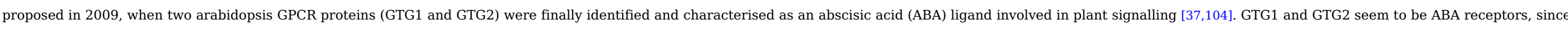



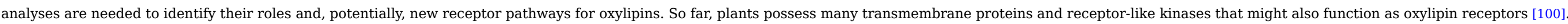

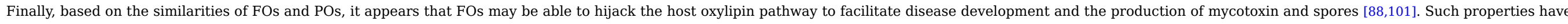

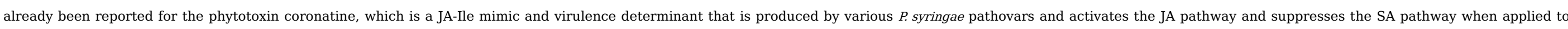
arabidopsis plants [49,89]. Recent studies have also shown the production of hydroxylated JA (11-OH-JA, 12-OH-JA), $N$-[(-)-jasmonoyl-(S)]-isoleucine, and other forms by different strains of fungi [106-108].

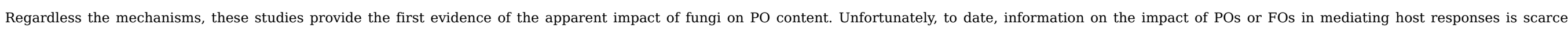

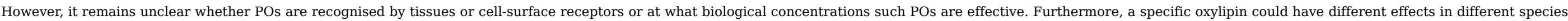
Currently, nothing is known about bacterial crosstalk and everything remains to be discovered in other pathosystems. 


\section{Concluding Remarks and Future Perspectives}

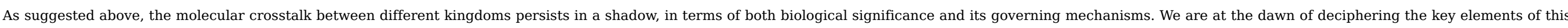





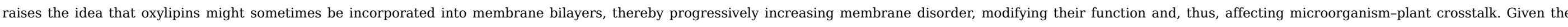

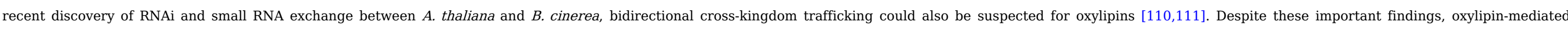
crosstalk between pathogens and host plants is a complex system that needs further study. Many questions remain to be answered, some of which have been neglected for years (see Outstanding Questions).

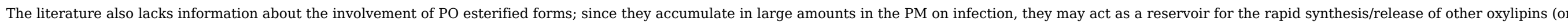



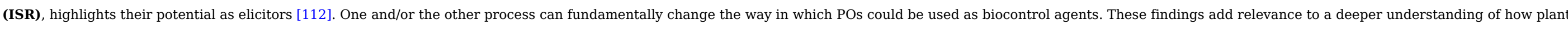

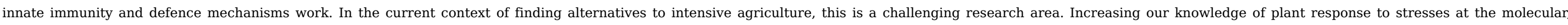
physiological, and metabolic levels will be vital for the development of new plant varieties and even more in developing new biopesticides.

Outstanding Questions

\section{What are the nonsignalling roles of phyto-oxylipins?}

Do oxylipins studied in vitro retain interesting antimicrobial properties in planta? What are their spectrum and mechanisms of action? Why do POs cause damage to the pathogen and not to the plant?

Are POs involved in innate immunity as elicitors or are they only direct biocides - or maybe both? Can POs be potential biocontrol agents?

Do plants use proteic receptors or equivalents for perception of oxylipins (POs or other oxylipins) or is it a mechanism independent of receptors (e.g., interaction with the lipidic fraction of the plant plasma membrane)?

How are POs, and more generally oxylipins, involved in interkingdom communication?

\section{Uncited references}

[113], [114]

\section{Acknowledgments}



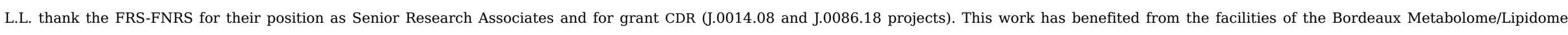
Facility-MetaboHUB (grant no. ANR-11-INBS-0010) to S.M. The authors are grateful to Manon Genva and Dr Caroline De Clerck for their interesting remarks and suggestions.

\section{References}

1. M. Barbosa, et al., Biologically active oxylipins from enzymatic and nonenzymatic routes in macroalgae, Mar. Drugs 14, $2016,23$.

2. G.A. Howe, Plant hormones: metabolic end run to jasmonate, Nat. Chem. Biol. 14, 2018, 109-110.

3. A.J. Koo, Metabolism of the plant hormone jasmonate: a sentinel for tissue damage and master regulator of stress response, Phytochem. Rev. 17, $2018,51-80$.

4. C. Wasternack and I. Feussner, The oxylipin pathways: biochemistry and function, Annu. Rev. Plant Biol. 69, 2018, 363-386.

5. E. Garreta-Lara, et al., Effect of psychiatric drugs on Daphnia magna oxylipin profiles, Sci. Total Environ. 644, 2018, 1101-1109. 
6. Q. Li, et al., Transporter-mediated nuclear entry of jasmonoyl-isoleucine is essential for jasmonate signaling, Mol. Plant 10, 2017, 695-708.

7. E.E. Farmer, et al., Jasmonates and related oxylipins in plant responses to pathogenesis and herbivory, Curr. Opin. Plant Biol. 6, 2003, 372-378.

8. I. Ponce de León, et al., Oxylipins in moss development and defense, Front. Plant Sci. 6, 2015, 483.

9. A.V. Ogorodnikova, et al., Oxylipins in the spikemoss Selaginella martensii: detection of divinyl ethers, 12-oxophytodienoic acid and related cyclopentenones, Phytochemistry 118, 2015, 42-50.

10. J. Lupette, et al., Non-enzymatic synthesis of bioactive isoprostanoids in the diatom Phaeodactylum following oxidative stress 1, Plant Physiol 178, $2018,1344-1357$.

11. G. Griffiths, Biosynthesis and analysis of plant oxylipins, Free Radic. Res. 49, 2015, 565-582.

12. T. Vellosillo, et al., Defense activated by 9-lipoxygenase-derived oxylipins requires specific mitochondrial proteins, Plant Physiol 161, $2013,617-627$.

13. E. Alméras, et al., Reactive electrophile species activate defense gene expression in Arabidopsis, Plant J 34, 2003, 205-216.

14. L. Mène-Saffrané, et al., Nonenzymatic oxidation of trienoic fatty acids contributes to reactive oxygen species management in Arabidopsis, J. Biol. Chem. 284, 2009, 1702-1708.

15. S. Mueller, et al., General detoxification and stress responses are mediated by oxidized lipids through TGA transcription factors in Arabidopsis, Plant Cell 20, $2008,768-785$.

16. F. Brodhun and I. Feussner, Oxylipins in fungi, FEBS J 278, 2011, 1047-1063.

17. G.J. Fischer and N.P. Keller, Production of cross-kingdom oxylipins by pathogenic fungi: an update on their role in development and pathogenicity, J. Microbiol. 54, 2016 , 254-264.

18. C. Wasternack and S. Song, Jasmonates: biosynthesis, metabolism, and signaling by proteins activating and repressing transcription, J. Exp. Bot. 68, $2017,1303-1321$.

19. M. Genva, et al., New insights into the biosynthesis of esterified oxylipins and their involvement in plant defense and developmental mechanisms, Phytochem. Rev. 8, 2019, 343-359.



21. T. Behl, et al., Role of leukotrienes in diabetic retinopathy, Prostaglandins Other Lipid Mediat 122, 2016, 1-9.

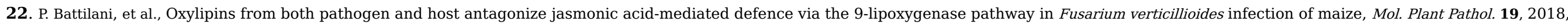
$2162-2176$.

23. S. Allmann, et al., Oxylipin channelling in Nicotiana attenuata: lipoxygenase 2 supplies substrates for green leaf volatile production, Plant Cell Environ 33, 2010 , $2028-2040$.

24. A. Chini, et al., An OPR3-independent pathway uses 4,5-didehydrojasmonate for jasmonate synthesis, Nat. Chem. Biol. 14, 2018, 171-178.

25. C. Böttcher and S. Pollmann, Plant oxylipins: plant responses to 12-oxo-phytodienoic acid are governed by its specific structural and functional properties, FEBS J 276, 2009 , 4693-4704.

26. A. Santino, et al., Jasmonate signaling in plant development and defense response to multiple (a)biotic stresses, Plant Cell Rep 32, 2013, 1085-1098.

27. Y. Sun, et al., The role of wheat jasmonic acid and ethylene pathways in response to Fusarium graminearum infection, Plant Growth Regul 80, $2016,69-77$.

28. L. Zhang, et al., Jasmonate signaling and manipulation by pathogens and insects, J. Exp. Bot 68, 2017, 1371-1385.

29. T. Heitz, et al., The rise and fall of jasmonate biological activities, Subcell. Biochem. 86, 2016, 405-426.

30. S. Fonseca, et al., (+)-7-iso-Jasmonoyl-L-isoleucine is the endogenous bioactive jasmonate, Nat. Chem. Biol. 5, 2009, 344-350.

31. P. Delaplace, et al., Oxylipin profile and antioxidant status of potato tubers during extended storage at room temperature, Plant Physiol. Biochem. 46, 2008, 1077-1084.

32. M.L. Fauconnier, et al., Changes in oxylipin synthesis after Phytophthora infestans infection of potato leaves do not correlate with resistance, Plant Physiol. Biochem. 46, 2008, 823-831. 
33. M.E. Ghanem, et al., Organ-dependent oxylipin signature in leaves and roots of salinized tomato plants (Solanum lycopersicum), J. Plant Physiol. 169, 2012 , 1090-1101.

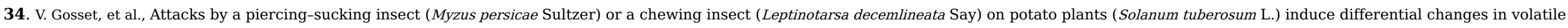
compound release and oxylipin synthesis, J. Exp. Bot. 60, 2009, 1231-1240.

35. G. Granér, et al., Screening of oxylipins for control of oilseed rape (Brassica napus) fungal pathogens, Phytochemistry 63, 2003, 89-95.

36. R.J. León Morcillo, et al., Plant 9-lox oxylipin metabolism in response to arbuscular mycorrhiza, Plant Signal. Behav. 7, 2012, 1584-1588.

37. D.I. Tsitsigiannis and N.P. Keller, Oxylipins as developmental and host-fungal communication signals, Trends Microbiol 15, 2007, $109-118$.

38. J. Vicente, et al., Role of 9-lipoxygenase and $\alpha$-dioxygenase oxylipin pathways as modulators of local and systemic defense, Mol. Plant 5, 2012, 914-928.

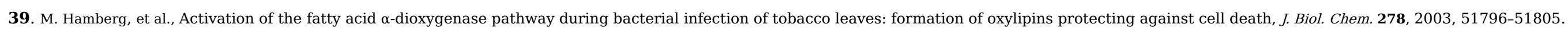

40. M. Mariutto, et al., Reprogramming of fatty acid and oxylipin synthesis in rhizobacteria-induced systemic resistance in tomato, Plant Mol. Biol. 84, 2014 , 455-467.



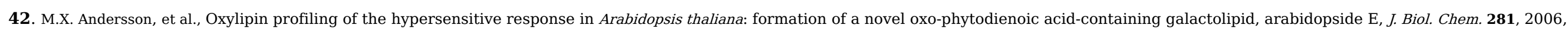
31528-31537.

43. J.D.G. Jones and J.L. Dangl, The plant immune system, Nature 444, 2006, 323-329.

44. W. Zhang, et al., Different pathogen defense strategies in Arabidopsis: more than pathogen recognition, Cells 7, 2018, E252.

45. G. Henry, et al., PAMPs, MAMPs, DAMPs and others: an update on the diversity of plant immunity elicitors, Biotechnol. Agron. Soc 16, $2012,12$.

46. J.S. Ramirez-Prado, et al., Plant immunity: from signaling to epigenetic control of defense, Trends Plant Sci 23, 2018, 833-844.

47. C. Chuberre, et al., Plant immunity is compartmentalized and specialized in roots, Front. Plant Sci. 9, 2018, 1692.

48. D. Tripathi, et al., Chemical elicitors of systemic acquired resistance - salicylic acid and its functional analogs, Curr. Plant Biol. 17, $2019,48-59$.

49. J.S. Thaler, et al., Evolution of jasmonate and salicylate signal crosstalk, Trends Plant SCi 17, 2012, 260-270.

50. M. Heyer, et al., A holistic approach to analyze systemic jasmonate accumulation in individual leaves of Arabidopsis rosettes upon wounding, Front. Plant Sci. 9, $2018,1569$.

51. G.Z. Han, Evolution of jasmonate biosynthesis and signalling mechanisms, J. Exp. Bot. 68, 2017, 1323-1331.

52. N.J. Atkinson and P.E. Urwin, The interaction of plant biotic and abiotic stresses: from genes to the field, J. Exp. Bot. 63, 2012, 3523-3544.

53. S.H. Chung, et al., Host plant species determines symbiotic bacterial community mediating suppression of plant defenses, Sci. Rep. 7, $2017,39690$.

54. Y. Hu, et al., Jasmonate regulates leaf senescence and tolerance to cold stress: crosstalk with other phytohormones, J. Exp. Bot. 68, 2017, $1361-1369$.

55. M. Hoffmann, et al., Auxin-oxylipin crosstalk: relationship of antagonists, J. Integr. Plant Biol. 53, 2011, 429-445.

56. J.E. Taylor, et al., Crosstalk between plant responses to pathogens and herbivores: a view from the outside in, J. Exp. Bot. 55, 2004, 159-168.

57. X. Di, et al., Involvement of salicylic acid, ethylene and jasmonic acid signalling pathways in the susceptibility of tomato to Fusarium oxysporum, Mol. Plant Pathol. 18, 2017, 1024-1035.

58. T. Lortzing and A. Steppuhn, Jasmonate signalling in plants shapes plant-insect interaction ecology, Curr. Opin. Insect Sci. 14, 2016, 32-39. 
59. A.L. Schilmiller and G.A. Howe, Systemic signaling in the wound response, Curr. Opin. Plant Biol. 8, 2005, 369-377.

60. C. Yan and D. Xie, Jasmonate in plant defence: sentinel or double agent?, Plant Biotechnol. J. 13, 2015, 1233-1240.

61. A.J.K. Koo, et al., A rapid wound signal activates the systemic synthesis of bioactive jasmonates in Arabidopsis, Plant J 59, 2009, 974-986.

62. W. Choi, et al., Rapid, long-distance electrical and calcium signaling in plants, Annu. Rev. Plant Biol. 67, 2016, 287-310.

63. W. Choi, et al., Orchestrating rapid long-distance signaling in plants with $\mathrm{Ca}^{2+}$, ROS and electrical signals, Plant J 90, $2018,698-707$.

64. S.A.R. Mousavi, et al., GLUTAMATE RECEPTOR-LIKE genes mediate leaf-to-leaf wound signalling, Nature 500, 2013, 422-426.

65. M. Toyota, et al., Glutamate triggers long-distance, calcium-based plant defense signaling, Science 6, 2018, 1112-1115.

66. T.C. Nguyen, et al., Identification of cell populations necessary for leaf-to- leaf electrical signaling in a wounded plant, Proc. Natl Acad. Sci. U. S. A. 115, 2018, 10178-10183.

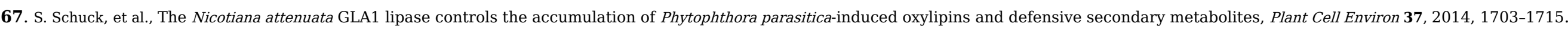

68. I. Prost, et al., Evaluation of the antimicrobial activities of plant oxylipins supports their involvement in defense against pathogens, Plant Physiol 139, $2005,1902-1913$.

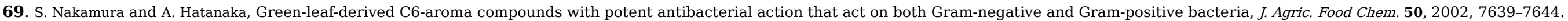

70. W. Ma, et al., Inhibitory effect of (E)-2-hexenal as a potential natural fumigant on Aspergillus flavus in stored peanut seeds, Ind. Crops Prod. 107, 2017, 206-210.

71. E.E. Farmer and M.J. Mueller, ROS-mediated lipid peroxidation and RES-activated signaling, Annu. Rev. Plant Biol. 64, 2013, 429-450.

72. S. Park, et al., Cyclophilin 20-3 relays a 12-oxo-phytodienoic acid signal during stress responsive regulation of cellular redox homeostasis, Proc. Natl Acad. Sci. U. S. A. 110, 2013, 9559-9564.

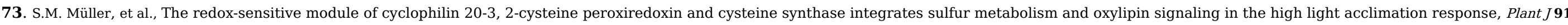
2017, 995-1014.

74. E.E. Farmer and C. Davoine, Reactive electrophile species, Curr. Opin. Plant Biol. 10, 2007, 380-386.

75. A.P. Desbois and V.J. Smith, Antibacterial free fatty acids: activities, mechanisms of action and biotechnological potential, Appl. Microbiol. Biotechnol. 85, 2010, 1629-1642.

76. J.J. Kabara, et al., Fatty acids and derivatives as antimicrobial agents, Antimicrob. Agents Chemother. 2, 1972, 23-28.

77. Y.Y. Toporkova, et al., Antimicrobial activity of geometric isomers of etherolenic acid - the products of plant lipoxygenase cascade, Biochem. Biophys. Mol. Biol. 480, 2018, 139-142.

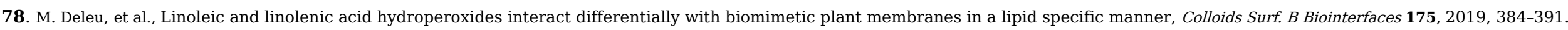

79. A. Sarwar, et al., Biocontrol activity of surfactin A purified from Bacillus NH-100 and NH-217 against rice bakanae disease, Microbiol. Res. 209, $2018,1-13$.

80. J. Deravel, et al., Mycosubtilin and surfactin are efficient, low ecotoxicity molecules for the biocontrol of lettuce downy mildew, Appl. Microbiol. Biotechnol. 98, 2014, 6255-6264.

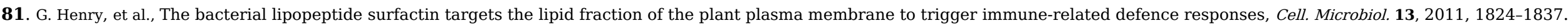

82. J. Gronnier, et al., Divide and rule: plant plasma membrane organization, Trends Plant Sci 23, 2018, 899-917.

83. M. Siebers, et al., Lipids in plant-microbe interactions, Biochim. Biophys. Acta 1861, 2016, 1379-1395.

84. M. Brodhagen, et al., Reciprocal oxylipin-mediated cross-talk in the Aspergillus-seed pathosystem, Mol. Microbiol. 67, 2008, 378-391.

85. Y. Okazaki and K. Saito, Roles of lipids as signaling molecules and mitigators during stress response in plants, Plant J 79, $2014,584-596$. 
86. A. Singh and M. Del Poeta, Lipid signalling in pathogenic fungi, Cell. Microbiol. 13, 2011, 177-185.

87. K. Sagini, et al., Extracellular vesicles as conveyors of membrane-derived bioactive lipids in immune system, Int. J. Mol. Sci. 19, 2018, E1227.

88. A. Kachroo and P. Kachroo, Fatty acid-derived signals in plant defense, Annu. Rev. Phytopathol. 47, 2009, 153-176.

89. J.W. Walley, et al., Fatty acids and early detection of pathogens, Curr. Opin. Plant Biol. 16, 2013, 520-526.

90. Y. Ishimaru, et al., GTR1 is a jasmonic acid and jasmonoyl-l-isoleucine transporter in Arabidopsis thaliana, Biosci. Biotechnol. Biochem. 8451, $2017,249-255$.

91. H. Saito, et al., The jasmonate-responsive GTR1 transporter is required for gibberellin-mediated stamen development in Arabidopsis, Nat. Commun. 6, 2015, 6095.

92. C.C. Yang and K.W. Chang, Eicosanoids and HB-EGF/EGFR in cancer, Cancer Metastasis Rev 37, 2018, 385-395.

93. D. Wang and R.N. Dubois, Eicosanoids and cancer, Nat. Rev. Cancer 10, 2010, 181-193.

94. J.A. Cornejo-García, et al., Pharmacogenomics of prostaglandin and leukotriene receptors, Front. Pharmacol. 7, $2016,316$.

95. M.C. Noverr, et al., Production of eicosanoids and other oxylipins by pathogenic eukaryotic microbes, Clin. Microbiol. Rev. 16, 2003, 517-533.

96. K.J. Affeldt, et al., Aspergillus oxylipin signaling and quorum sensing pathways depend on G protein-coupled receptors, Toxins (Basel) 4, 2012, 695-717.

97. K.J. Affeldt, et al., Global survey of canonical Aspergillus flavus GPCRs, MBio 5, 2014, e01501-e01514.

98. Z. Raviv, et al., The anti-cancer activities of jasmonates, Cancer Chemother. Pharmacol. 71, 2013, 275-285.

99. T. Savchenko, et al., Arachidonic acid: an evolutionarily conserved signaling molecule modulates plant stress signaling networks, Plant Cell 22, $2010,3193-3205$.

100. S.A. Christensen and M.V. Kolomiets, The lipid language of plant-fungal interactions, Fungal Genet. Biol. 48, 2011, 4-14.

101. X. Gao and M.V. Kolomiets, Host-derived lipids and oxylipins are crucial signals in modulating mycotoxin production by fungi, Toxin Rev 28, 2009 , $79-88$.

102. D.I. Tsitsigiannis, et al., Aspergillus infection inhibits the expression of peanut 13S-HPODE-forming seed lipoxygenases, Mol. Plant Microbe Interact. 18, 2005, $1081-1089$.

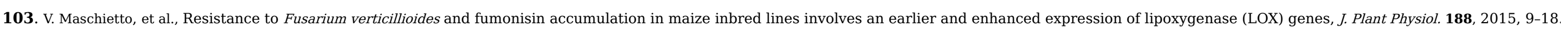

104. S. Pandey, et al., Two novel GPCR-type G proteins are abscisic acid receptors in Arabidopsis, Cell 136, 2009, 136-148.

105. J.M. Risk, et al., Reevaluation of abscisic acid-binding assays shows that G-protein-coupled receptor2 does not bind abscisic acid, Plant Physiol 150, 2009 , 6-11.

106. A. Chini, et al., The fungal phytotoxin lasiojasmonate A activates the plant jasmonic acid pathway, J. Exp. Bot. 69, 2018, 3095-3102.

107. E. Chanclud and J. Morel, Plant hormones : a fungal point of view, Mol. Plant Pathol. 17, 2016, 1289-1297.

108. R.N. Patkar, et al., A fungal monooxygenase-derived jasmonate attenuates host innate immunity, Nat. Chem. Biol. 11, 2015, 733-740.

109. H. Tran Thanh, et al., Toxicity of fatty acid hydroperoxides towards Yarrowia lipolytica: implication of their membrane fluidizing action, Biochim. Biophys. Acta 1768, 2007, 2256-2262.

110. M. Wang, et al., Bidirectional cross-kingdom RNAi and fungal uptake of external RNAs confer plant protection, Nat. Plants 2, $2017,16151$.

111. A. Weiberg, et al., Fungal small RNAs suppress plant immunity by hijacking host RNA interference pathways, Science 342, 2014, 118-123.

112. M. Ongena, et al., Surfactin and fengycin lipopeptides of Bacillus subtilis as elicitors of induced systemic resistance in plants, Environ. Microbiol. 9, 2007, 1084-1090.

113. E. Blée, Impact of phyto-oxylipins in plant defense, Trends Plant Sci 7, 2002, 315-321. 
114. T.V. Savchenko, et al., Oxylipins and plant abiotic stress resistance, Biochemistry (Mosc.) 79, 2014, 362-375.

\section{Glossary}

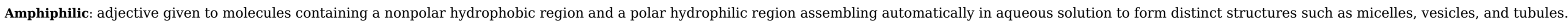

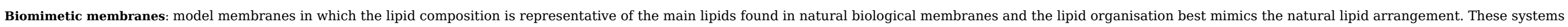

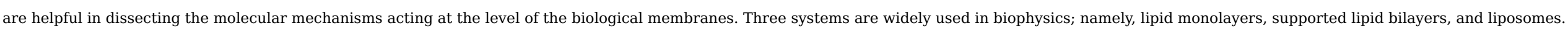
Crosstalk: a term used when a common cellular component is engaged in more than one signal transduction chain and allows information exchange between different signalling pathways. Elicitors: natural or synthetic compounds that are exogenously applied and induce defence responses in plants similar to those induced by pathogen infections.

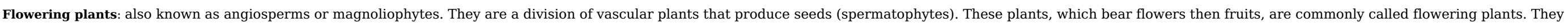
include dicotyledons and monocotyledons.

G protein-coupled receptors (GPCRs): cell type-specific transmembrane proteic receptors detecting external signals and transmitting them into the cell to induce various responses.

Induced systemic resistance (ISR): strengthening of the defence capacity of the entire plant against a broad spectrum of pathogens; acquired during local induction by beneficial microbes.


Oxylipin: large class of lipid metabolites derived from the oxidation of PUFAs.

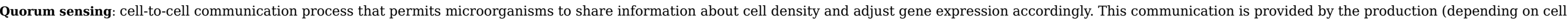
density) and release of chemical signal molecules.

Systemic acquired resistance (SAR): strengthening of the defence capacity of the entire plant against a broad spectrum of pathogens; acquired after a primary local pathogen infection.

Keywords: oxylipins; lipoxygenase pathway; plant-pathogen interactions; membrane interactions; interkingdom communication

\section{Highlights}

Many studies have shown that specific oxylipin signatures are shaped during (a)biotic stresses

It is generally accepted that divinyl-, keto-, and hydroxy-fatty acids and fatty acid hydroperoxides exhibit strong direct antimicrobial activities, whereas the roles of jasmonic acid and some volatile aldehydes seem to be related to signalling activities only.

Oxylipins' chemical structures are related to their biological activities.

Current studies show that the lipid composition of the plasma membrane has important roles in the interaction of plant oxylipins with plant cells.

It is becoming clear that many organisms use the oxylipin pathways as a common process for interkingdom communication

\section{Queries and Answers}


appropriate places in the text. (They were not cited in the manuscript.)

Answer: Indeed, ref 113 and 114 can be removed from the list as they are not used in the manuscript at the end. Sorry for the mistake. 